\title{
ADUBAÇÃO FOLIAR COM MICRONUTRIENTES EM ARROZ IRRIGADO, EM ÁREA SISTEMATIZADA
}

\author{
SPRAY APPLICATION OF MICRONUTRIENTS ON IRRIGATED RICE CULTIVATED \\ IN LEVELED SOIL
}

\section{Enio Marchezan ${ }^{1}$ Osmar Santos dos Santos ${ }^{2}$ Luis Antonio de Avila ${ }^{3}$ Ricardo Posser da Silva $^{4}$}

\section{RESUMO}

O objetivo deste trabalho foi avaliar o efeito da aplicação de micronutrientes em arroz irrigado, em área de várzea após a sistematização. Foi realizado durante três anos agrícolas em PLANOSSOLO Hidromórfico eutrófico arênico, instalado em local de onde foi retirada uma camada de solo de cerca de $30 \mathrm{~cm}$ de profundidade. A adubação com $N, P$ e $K$ foi realizada de acordo com a recomendação técnica para a cultura. o delineamento experimental foi de blocos ao acaso com quatro repetições, constituído de um tratamento denominado "completo", contendo os micronutrientes boro $\left(\mathrm{H}_{3} \mathrm{BO}_{3}\right)$, cobre $\left(\mathrm{CuSO}_{4}\right)$, ferro $\left(\mathrm{FeSO}_{4}\right)$, manganês $\left(\mathrm{MnCl}_{2}\right)$, molibdênio $\left(\mathrm{Na}_{2} \mathrm{MoO}_{4}\right)$ e zinco $\left(\mathrm{ZnSO}_{4}\right)$ e de seis outros formados pela omissão de um micronutriente de cada vez, além de testemunha sem micronutrientes. Em 1996/97, foram aplicados $70 \mathrm{~g} \mathrm{ha} a^{-1} \mathrm{Zn}$, $70 \mathrm{~g} \mathrm{ha} \mathrm{C}^{-1} \mathrm{Mn}, 40 \mathrm{gha^{-1 }} \mathrm{Fe}, 50 \mathrm{gha^{-1 }}$ B, 12,5g ha- Cu e $4 \mathrm{~g} \mathrm{ha} \mathrm{g}^{-1} \mathrm{Mo}$, via foliar, no perfilhamento e no início da formação do primórdio floral das plantas de arroz. Em 1997/98, foram utilizadas as mesmas soluções, porém com aplicação apenas no perfilhamento. Em 1998/99, as doses dos micronutrientes foram alteradas para

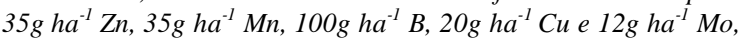
aplicados no perfilhamento, não sendo utilizado o ferro no tratamento completo nem na composição dos demais tratamentos. A sistematização da área causou redução no teor de matéria orgânica, macro e micronutrientes, exceto o boro e aumento de alumínio no solo. Verificou-se que não houve efeito da aplicação foliar de micronutrientes no rendimento de grãos de arroz irrigado.

Palavras-chave: várzea, Oryza sativa, adubação.

\section{SUMMARY}

The objective of this experiment was to evaluate the effect of micronutrient application on irrigated rice in an area previously leveled. The experiment was carried out during three

\begin{abstract}
years on a Albalqualf soil in which the soil surface layer of about $30 \mathrm{~cm}$ had been removed. NPK fertilization was applied according to soil analysis. The experiment design was a randomized block with four replications. The "complete" treatment had the micronutrients boron $\left(\mathrm{H}_{3} \mathrm{BO}_{3}\right)$, copper $\left(\mathrm{CuSO}_{4}\right)$, iron $\left(\mathrm{FeSO}_{4}\right)$, manganese $\left(\mathrm{MnCl}_{2}\right)$, molybdenum $\left(\mathrm{Na}_{2} \mathrm{MoO}_{4}\right)$ and zinc $\left(\mathrm{ZnSO}_{4}\right)$ and treatments in which each one of the micronutrients was omitted besides a check without micronutrients. In the 1996/97 growing season the following rates were applied as leaf spraying at tillering and begining of flowering differentiation stage: $\mathrm{Zn}$

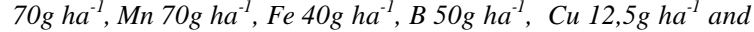
Mo $4 \mathrm{~g} \mathrm{ha}^{-1}$. In the 1997/98 growing season the same solutions were used but only at tillering. In 1998/99 the micronutrients doses were altered to $\mathrm{Zn} 35 \mathrm{~g} \mathrm{ha}^{-1}, \mathrm{Mn} 35 \mathrm{~g} \mathrm{ha}^{-1}, \mathrm{~B} 100 \mathrm{~g} \mathrm{ha}{ }^{-1}, \mathrm{Cu}$ $20 \mathrm{~g} \mathrm{ha}^{-1}$ and Mol2 $\mathrm{g} \mathrm{ha}^{-1}$ at tillering growth stage and $\mathrm{Fe}$ no longer was used. Soil leveling decreased soil organic matter, macro and micronutrients except boron and increased aluminum levels in the soil. It was observed that leaf spraying application of micronutrients on leaves did not affect seed yield in any of the years in which the experiments were conducted.
\end{abstract}

Key words: lowland, Oryza sativa, fertilizer.

\section{INTRODUÇÃO}

A orizicultura é uma atividade agrícola importante para a economia do Brasil e especialmente do Rio Grande do Sul. Cerca de cem municípios que compõem a metade sul do Rio Grande do Sul têm no arroz seu principal produto agrícola e, segundo SEBRAE (1999), a adequação das áreas de várzea, através de sistematização, possibilita a realização de uma lavoura mais intensiva, diversificada e sustentável.

\footnotetext{
${ }^{1}$ Engenheiro Agrônomo, Doutor, Professor Titular, Departamento de Fitotecnia (DF), Centro de Ciências Rurais (CCR), Universidade Federal de Santa Maria (UFSM), 97.105-900. E-mail: emarch@ccr.ufsm.br. Autor para correspondência.

${ }^{2}$ Engenheiro Agrônomo, Doutor, Professor Titular, Pesquisador do CNPq, DF, CCR, UFSM.

${ }^{3}$ Engenheiro Agrônomo, MSc., Professor Assistente, DF, CCR, UFSM.

${ }^{4}$ Aluno do curso de Agronomia, UFSM.
} 
A sistematização da área, que inclui o nivelamento da superfície do terreno, permite que possam ser utilizados sistemas de cultivo de arroz como o pré-germinado, o mix de pré-germinado e o transplante de mudas. Esta prática beneficia a irrigação também nos sistemas de cultivo convencional e plantio direto.

O nivelamento das áreas, executado durante o processo de sistematização, provoca desuniformidade nas características físico-químicas do solo entre as áreas de corte e aterro, especialmente nos primeiros anos de cultivo. Os reflexos na produtividade ocorrem com intensidade variável de acordo com o tipo de solo, processo utilizado para o nivelamento dos talhões $\mathrm{e}$ profundidade de mobilização do solo, entre outros fatores (MARCHEZAN et al., 1999). Esta situação adquire maior importância devido ao crescente aumento de áreas sistematizadas no Rio Grande do Sul e suscita questionamentos a respeito da recuperação da produtividade nas áreas de corte através da aplicação diferencial de nutrientes. A mobilização do solo realizada durante o processo de nivelamento da área para sistematização provoca algumas alterações na concentração de macro e micronutrientes na região do sistema radicular (REZER et al., 1997), pois a remoção dos $30 \mathrm{~cm}$ da superfície do solo reduz os teores dos nutrientes em cerca de $50 \%$, incluindo a matéria orgânica.

A possibilidade de elevar a produtividade da lavoura com a adição de micronutrientes na cultura do arroz foi estudada por diversos pesquisadores (PAULA $\boldsymbol{e t}$ al., 1991, ANDRADE $\boldsymbol{e} \boldsymbol{t}$ $\boldsymbol{a l} .$, 1997, REZER $\boldsymbol{e t} \boldsymbol{a l} .$, 1997, ANDRADE $\boldsymbol{e} \boldsymbol{t} \boldsymbol{a l}$., 1998 e DYNIA $\boldsymbol{e} \boldsymbol{t} \boldsymbol{a l} .$, 1998) que não encontraram incrementos de produtividade. Por outro lado, autores como BARBOSA FILHO et al. (1983), LOPES et al. (1984), BARBOSA FILHO $\boldsymbol{e t}$ al. (1990) e PAULA et al. (1991) obtiveram acréscimos de produtividade com a aplicação de $\mathrm{Zn}$ em arroz. Estes diferentes resultados estão associados ao tipo de solo e ao modo de cultivo do arroz (GALRÃO $\boldsymbol{e t}$ al., 1984) pois, segundo DECHEN et al. (1991), em solos arenosos pode ocorrer com mais freqüência a deficiência de micronutrientes que, em áreas sistematizadas, dependendo da profundidade de remoção de solo, possibilita resposta positiva à adubação.

São poucos os dados de pesquisa a respeito do efeito da aplicação de micronutrientes em arroz cultivado em área sistematizada e, em vista disso, foi realizado este estudo, durante três anos agrícolas, com o objetivo de avaliar o efeito da aplicação foliar de micronutrientes em arroz irrigado, cultivado em várzea após a sistematização.

\section{MATERIAL E MÉTODOS}

Os experimentos foram conduzidos em área experimental da Universidade Federal de Santa Maria (UFSM), em solo PLANOSSOLO Hidromórfico eutrófico arênico (EMBRAPA, 1999) pertencente à unidade de mapeamento Vacacaí. Foram instalados em área que sofreu processo de nivelamento superficial e sistematização, em local de onde foi retirada uma camada de solo de cerca de $30 \mathrm{~cm}$ de profundidade, conhecida como área de "corte" no processo de sistematização. Esses experimentos, em 1996/97 e 1997/98, foram implantados em área de primeiro ano após a sistematização e, em 1998/99, em área sistematizada, que foi cultivada anteriormente com arroz irrigado por um ano agrícola, em talhões contíguos, separados apenas por marachas ou taipas.

No primeiro ano agrícola, procedeu-se a análise química do solo onde foram instalados os experimentos, sendo amostrado o mesmo local, antes e depois da execução do nivelamento da área (Tabela 1).

A adubação, nos três anos de cultivo, foi feita de acordo com a interpretação da análise química do solo, seguindo sugestões de adubação contidas em IRGA (1995) e consistiu de $12 \mathrm{~kg} \mathrm{ha}^{-1}$ de nitrogênio, $50 \mathrm{~kg} \mathrm{ha}^{-1}$ de $\mathrm{P}_{2} \mathrm{O}_{5}$ e $50 \mathrm{~kg} \mathrm{ha}^{-1}$ de $\mathrm{K}_{2} \mathrm{O}$. Utilizou-se sistema convencional de cultivo, com a cultivar EMBRAPA 7 - Taim em 1996/97 e IRGA 417, nos anos agrícolas de 1997/98 e 1998/99. A adubação de cobertura foi realizada no perfilhamento, na dose de $40 \mathrm{~kg} \mathrm{ha}^{-1}$ de nitrogênio.

O delineamento experimental foi de blocos ao acaso com quatro repetições, constituído

Tabela 1 - Características físico-químicas do solo, antes e após a sistematização da área de várzea em 1996/97. Santa Maria, RS. 2000.

\begin{tabular}{lrc}
\hline Componentes & Antes da sistematização & Após a sistematização \\
\hline Argila $(\%)$ & 25,50 & 19,00 \\
pH $\left(\mathrm{H}_{2} \mathrm{O}\right)$ & 4,65 & 4,75 \\
$\mathrm{pH}(\mathrm{SMP})$ & 5,70 & 5,40 \\
$\mathrm{P}(\mathrm{mg} / \ell)$ & 4,20 & 2,00 \\
$\mathrm{~K}(\mathrm{mg} / \ell)$ & 38,00 & 21,00 \\
$\mathrm{M} . \mathrm{O} .(\%)$ & 2,15 & 0,95 \\
$\mathrm{Al}\left(\mathrm{cmol}_{\mathrm{c}} / \ell\right)$ & 0,95 & 1,70 \\
$\mathrm{Ca}\left(\mathrm{cmol}_{\mathrm{c}} / \ell\right)$ & 4,35 & 1,05 \\
$\mathrm{Mg}\left(\mathrm{cmol}_{\mathrm{c}} / \ell\right)$ & 1,25 & 0,30 \\
$\mathrm{CTC}\left(\mathrm{cmol}_{\mathrm{c}} / \ell\right)$ & 8,10 & 4,60 \\
$\mathrm{H}+\mathrm{Al}\left(\mathrm{cmol}_{\mathrm{c}} / \ell\right)$ & 2,35 & 3,20 \\
$\%$ Sat. Bases & 70,40 & 30,10 \\
$\%$ Sat. Al & 11,70 & 35,40 \\
$\mathrm{Zn}\left(\mathrm{cmol}_{\mathrm{c}} / \ell\right)$ & 0,90 & 0,15 \\
$\mathrm{Cu}\left(\mathrm{cmol}_{\mathrm{c}} / \ell\right)$ & 1,15 & 0,55 \\
$\mathrm{~B}\left(\mathrm{cmol}_{\mathrm{c}} / \ell\right)$ & 0,30 & 0,30 \\
$\mathrm{Mn}\left(\mathrm{cmol}_{\mathrm{c}} / \ell\right)$ & 26,00 & 2,00 \\
\hline
\end{tabular}


de um tratamento denominado "completo", contendo os micronutrientes boro $\left(\mathrm{H}_{3} \mathrm{BO}_{3}\right)$, cobre $\left(\mathrm{CuSO}_{4}\right)$, ferro $\left(\mathrm{FeSO}_{4}\right)$, manganês $\left(\mathrm{MnCl}_{2}\right)$, molibdênio $\left(\mathrm{Na}_{2} \mathrm{MoO}_{4}\right)$ e zinco $\left(\mathrm{ZnSO}_{4}\right)$ e de seis outros, formados pela omissão de um micronutriente de cada vez, além de testemunha sem micronutrientes. Em 1996/97, foram aplicados $70 \mathrm{~g} \mathrm{ha}^{-1} \mathrm{Zn}, 70 \mathrm{~g} \mathrm{ha}^{-1}$ Mn, 40g ha ${ }^{-1} \mathrm{Fe}, 50 \mathrm{~g} \mathrm{ha}^{-1} \mathrm{~B}, 12,5 \mathrm{~g} \mathrm{ha}^{-1} \mathrm{Cu}$ e $4 \mathrm{~g} \mathrm{ha}^{-1}$ Mo, via foliar, no perfilhamento e no início da formação do primórdio floral das plantas de arroz. Em 1997/98, foram utilizadas as mesmas soluções, porém com aplicação apenas no perfilhamento. Em 1998/99, as doses dos micronutrientes foram alteradas para $35 \mathrm{~g} \mathrm{ha}^{-1} \mathrm{Zn}, 35 \mathrm{~g} \mathrm{ha}^{-1} \mathrm{Mn}, 100 \mathrm{~g} \mathrm{ha}^{-1} \mathrm{~B}$, $20 \mathrm{~g} \mathrm{ha}{ }^{-1} \mathrm{Cu}$ e $12 \mathrm{~g}$ ha $^{-1}$ Mo, aplicados no perfilhamento. Neste ano não se utilizou o ferro no tratamento completo nem na composição dos demais tratamentos.

As parcelas foram compostas de quinze linhas de $6,0 \mathrm{~m}$ de comprimento, espaçadas de $0,20 \mathrm{~m}$. As pulverizações foram efetuadas com pulverizador costal pressurizado a $\mathrm{CO}_{2}$, utilizandose vazão equivalente a $150 \ell \mathrm{ha}^{-1}$ da solução com micronutrientes. A barra pulverizadora abrangia uma faixa de cobertura de $2,5 \mathrm{~m}$ e a área útil da parcela utilizada para a estimativa do rendimento de grãos constituiu-se de oito linhas centrais com $5,0 \mathrm{~m}$ de comprimento, correspondendo a $8,0 \mathrm{~m}^{2}$.

O controle de plantas daninhas foi realizado com aplicação do herbicida quinclorac na dose de $375 \mathrm{~g}$ de i.a.ha ${ }^{-1}$, cerca de 15 dias após a emergência do arroz.

A irrigação por inundação foi estabelecida aos 20 dias após a emergência do arroz, com lâmina de água de cerca de $10 \mathrm{~cm}$, de forma contínua, até a colheita. Não foi necessário realizar controle de pragas e nem de moléstias.

Foram avaliados o rendimento de grãos, corrigindo a massa de grãos para $13 \%$ de umidade e a renda do beneficiamento, quantificando grãos inteiros e quebrados. A massa de 1.000 grãos e a esterilidade de espiguetas foram determinadas em 10 panículas coletadas ao acaso por parcela, separando-se as espiguetas cheias das vazias. A estatura de plantas foi obtida pela medida de 10 plantas ao acaso em cada parcela, tomada do solo até a extremidade da panícula. Os resultados foram submetidos à análise de variância e as médias comparadas pelo teste de Duncan ao nível de $5 \%$ de probabilidade de erro.

\section{RESULTADOS E DISCUSSÃO}

As tabelas 2, 3 e 4 contêm os resultados das avaliações realizadas em
Tabela 2 - Rendimento de grãos, estatura de plantas, massa de 1.000 grãos (MMG) e percentagem de grãos inteiros de arroz irrigado, cultivar EMBRAPA 7 - Taim, com aplicação foliar de micronutrientes, ano 1996/97. Santa Maria, RS. 2000.

\begin{tabular}{lcccc}
\hline Tratamento & $\begin{array}{c}\text { Rendimento } \\
\left(\mathrm{kg} \mathrm{ha}^{-1}\right)\end{array}$ & $\begin{array}{c}\text { Estatura } \\
(\mathrm{cm})\end{array}$ & $\begin{array}{c}\text { MMG } \\
(\mathrm{g})\end{array}$ & $\begin{array}{c}\text { Grãos inteiros } \\
(\%)\end{array}$ \\
\hline Testemunha & $5.484^{\mathrm{ns}}$ & $71^{\mathrm{ns}}$ & $27^{\mathrm{ns}}$ & $61^{\mathrm{ns}}$ \\
Menos B & 5.555 & 72 & 26 & 62 \\
Menos Cu & 5.472 & 72 & 26 & 62 \\
Menos Fe & 5.691 & 73 & 26 & 62 \\
Menos Mn & 5.744 & 73 & 26 & 61 \\
Menos Mo & 5.695 & 74 & 26 & 63 \\
Menos Zn & 5.923 & 72 & 27 & 64 \\
Completo & 5.565 & 73 & 26 & 62 \\
Média & 5.641 & 73 & 26 & 62 \\
C.V. $(\%)$ & 6,37 & 3,18 & 1,61 & 3,35 \\
\hline
\end{tabular}

${ }^{\text {ns }}$ Teste F não significativo em nível de 5\% de probabilidade.

1996/97, 1997/98 e 1998/99, respectivamente. Verifica-se que não houve efeito da aplicação foliar de nenhum dos tratamentos com micronutrientes na produção de arroz irrigado, nos três anos de realização dos experimentos, concordando com os resultados de PAULA $\boldsymbol{e} \boldsymbol{t}$ al. (1991), ANDRADE $\boldsymbol{e} \boldsymbol{t}$ al. (1997), REZER $\boldsymbol{e t}$ al. (1997), ANDRADE $\boldsymbol{e t} \boldsymbol{a l}$. (1998) e DYNIA et al. (1998). A produtividade média mais baixa, obtida nos dois primeiros anos, pode ser explicada, em parte, pelo fato de os experimentos terem sido implantados no primeiro ano após a realização da sistematização da área, enquanto que, em 1998/99, foi instalado em área que já havia sido cultivada com arroz irrigado por um ano. Além disso, condições de clima devem ter

Tabela 3 - Rendimento de grãos, estatura de plantas, esterilidade de espiguetas, massa de 1.000 grãos (MMG) e percentagem de grãos inteiros de arroz irrigado, cultivar IRGA 417, com aplicação foliar de micronutrientes, ano 1997/98. Santa Maria, RS. 2000.

\begin{tabular}{lccccc}
\hline Tratamento & $\begin{array}{c}\text { Rendimento } \\
\left(\mathrm{kg} \mathrm{ha}^{-1}\right)\end{array}$ & $\begin{array}{c}\text { Estatura } \\
(\mathrm{cm})\end{array}$ & $\begin{array}{c}\text { Esterilidade } \\
(\%)\end{array}$ & $\begin{array}{c}\text { MMG } \\
(\mathrm{g})\end{array}$ & $\begin{array}{c}\text { Grãos inteiros } \\
(\%)\end{array}$ \\
\hline Testemunha & $4.526^{\mathrm{ns}}$ & $68 \mathrm{~b}^{*}$ & $22^{\mathrm{ns}}$ & $23,4 \mathrm{ab}$ & $64 \mathrm{ab}$ \\
Menos B & 4.681 & $74 \mathrm{ab}$ & 22 & $24,0 \mathrm{a}$ & $63 \mathrm{ab}$ \\
Menos Cu & 4.460 & $70 \mathrm{ab}$ & 22 & $23,9 \mathrm{ab}$ & $63 \mathrm{ab}$ \\
Menos Fe & 4.900 & $72 \mathrm{ab}$ & 19 & $24,0 \mathrm{a}$ & $65 \mathrm{a}$ \\
Menos Mn & 4.785 & $70 \mathrm{ab}$ & 20 & $23,2 \mathrm{~b}$ & $63 \mathrm{ab}$ \\
Menos Mo & 4.921 & $73 \mathrm{ab}$ & 22 & $23,6 \mathrm{ab}$ & $65 \mathrm{a}$ \\
Menos Zn & 4.837 & $74 \mathrm{a}$ & 23 & $23,8 \mathrm{ab}$ & $61 \mathrm{~b}$ \\
Completo & 4.654 & $72 \mathrm{ab}$ & 21 & $23,8 \mathrm{ab}$ & $64 \mathrm{ab}$ \\
Média & 4.721 & 72 & 21 & 23,7 & 64 \\
C.V. $(\%)$ & 7,97 & 5,16 & 15,8 & 1,81 & 3,09 \\
\hline
\end{tabular}

${ }^{n s}$ Teste $\mathrm{F}$ não significativo em nível de $5 \%$ de probabilidade.

* Média não ligadas pela mesma letra diferem pelo teste de Duncan a $5 \%$ de probabilidade. 
Tabela 4 - Rendimento de grãos, estatura de plantas, esterilidade de espiguetas, massa de 1.000 grãos (MMG) e percentagem de grãos inteiros de arroz irrigado, cultivar IRGA 417, com aplicação foliar de micronutrientes, ano 1998/99. Santa Maria, RS. 2000.

\begin{tabular}{lccccc}
\hline Tratamento & $\begin{array}{c}\text { Rendimento } \\
\left(\mathrm{kg.ha}^{-1}\right)\end{array}$ & $\begin{array}{c}\text { Estatura } \\
(\mathrm{cm})\end{array}$ & $\begin{array}{c}\text { Esterilidade } \\
(\%)\end{array}$ & $\begin{array}{c}\text { MMG } \\
(\mathrm{g})\end{array}$ & $\begin{array}{c}\text { Grãos inteiros } \\
(\%)\end{array}$ \\
\hline Testemunha & $7.699^{\mathrm{ns}}$ & $81^{\mathrm{ns}}$ & $4,8 \mathrm{ab} *$ & $25,2 \mathrm{ab}$ & $64,3 \mathrm{c}$ \\
Menos B & 7.778 & 81 & $3,9 \mathrm{~b}$ & $25,6 \mathrm{ab}$ & $65,6 \mathrm{ab}$ \\
Menos Cu & 7.823 & 81 & $4,7 \mathrm{ab}$ & $25,8 \mathrm{ab}$ & $65,5 \mathrm{ab}$ \\
Menos Mn & 7.662 & 80 & $4,4 \mathrm{ab}$ & $24,8 \mathrm{~b}$ & $64,7 \mathrm{bc}$ \\
Menos Mo & 7.928 & 80 & $3,8 \mathrm{~b}$ & $25,6 \mathrm{ab}$ & $64,8 \mathrm{abc}$ \\
Menos Zn & 8.051 & 81 & $6,0 \mathrm{a}$ & $26,2 \mathrm{a}$ & $65,0 \mathrm{abc}$ \\
Completo & 7.873 & 82 & $4,4 \mathrm{ab}$ & $25,0 \mathrm{~b}$ & $65,7 \mathrm{a}$ \\
Média & 7.830 & 81 & 4,6 & 25,4 & 65,2 \\
C.V. $(\%)$ & 9,74 & 4,45 & 10,97 & 2,52 & 0,63 \\
\hline
\end{tabular}

${ }^{\text {ns }}$ Teste F não significativo em nível de 5\% de probabilidade.

* Média não ligadas pela mesma letra diferem pelo teste de Duncan a $5 \%$ de probabilidade.

influenciado pois, na tabela 4 , verifica-se que a esterilidade de espiguetas foi de apenas 4,3\%, enquanto, no ano anterior (Tabela 3), este mesmo parâmetro foi de $21,3 \%$, caracterizando condições desfavoráveis à produção de grãos.

Os resultados do primeiro ano, expresso na tabela 2 , revelam que não houve influência diferenciada da aplicação de micronutrientes para nenhuma das características avaliadas. No segundo ano (Tabela 3), os resultados mostram que os tratamentos com micronutrientes afetaram a estatura de planta, massa de 1.000 grãos e a quantidade de grãos inteiros. Já no terceiro ano (Tabela 4), verifica-se que a aplicação de micronutrientes afetou significativamente a esterilidade de espiguetas, a massa de 1.000 grãos e a percentagem de grãos inteiros.

No entanto, as diferenças observadas, foram de pequena amplitude, de modo que não repercutiram na produção de grãos, em nenhum dos anos de execução do experimento, apesar da sistematização da área ter promovido redução de macro e micronutrientes do solo (Tabela 1). Quando se compara esses valores com referenciais relatados na Recomendação de adubação e de calagem para os estados do Rio Grande do Sul e de Santa Catarina (COMISSÃO ..., 1995) contata-se que os elementos $\mathrm{Cu}, \mathrm{B}$ e $\mathrm{Mn}$ situam-se numa faixa de suficiência nutricional e apenas o Zn encontrava-se em nível considerado baixo.

Quanto à estatura de plantas e qualidade física de grãos, expressas pela quantidade de grãos inteiros, em resposta à aplicação de $\mathrm{Zn}$, os resultados foram divergentes se comparados os três anos, pois em 1996/97 (Tabela 2), o micronutriente afetou positivamente a estatura de planta, mas reduziu a quantidade de grãos inteiros. Em 1998/99 (Tabela 4), o Zn não afetou a estatura de plantas, mas influenciou na elevação do número de grãos inteiros. De forma semelhante, BARBOSA FILHO et al. (1983) constataram aumento na estatura de plantas, enquanto ANDRADE $\boldsymbol{e t}$ al. (1998) não observaram elevação de estatura de plantas com aplicação de Zn.

Interpretação semelhante pode ser feita se analisada a aplicação do tratamento completo com micronutrientes ou com a supressão de determinado micronutriente. Os resultados divergentes à aplicação de micronutrientes encontrados na literatura estão relacionados ao tipo de solo onde são instalados os experimentos, bem como as diferenças de condições de clima verificadas de local para local, entre anos de avaliação. Além disso, Boareto \& Roselen, citados por REZER et al. (1997) relatam que a resposta a aplicação de micronutrientes está relacionada ao nível de produtividade obtido. Os autores consideram importante a utilização de micronutrientes quando se planeja produtividade superior a $6.000 \mathrm{~kg} \cdot \mathrm{ha}^{-1}$. Esta constatação não foi comprovada no presente trabalho, pois não houve resposta positiva quando se produziu menos de $5.000 \mathrm{~kg} \cdot \mathrm{ha}^{-1}$ (Tabela 3) e nem quando se atingiu produtividade próximas a $8.000 \mathrm{~kg} \mathrm{ha}^{-1}$ (Tabela 4).

\section{CONCLUSÃO}

A aplicação de micronutrientes, via foliar, não influenciou a produção de grãos do arroz irrigado cultivado em área de retirada de solo devido ao processo de nivelamento.

\section{REFERÊNCIAS BIBLIOGRÁFICAS}

ANDRADE, W.E. de B., SOUZA, A.F. de, CARVALHO, J.G. Limitações nutricionais para a cultura do arroz irrigado em solo orgânico da região Nordeste Fluminense. R Bras Ci Solo, Campinas, v.21, n.3, p.513-517, 1997.

ANDRADE, W.E. de B., SOUZA, A.F. de, CARVALHO, J.G. Deficiências nutricionais no arroz irrigado em sucessão ao feijoeiro em solo de várzea. Pesq Agropec Bras, Brasília, v.33, n.7, p.1129-1135, 1998.

BARBOSA FILHO, M.P., FAGERIA, N.K, FONSECA, J.R Tratamento de sementes de arroz com micronutrientes sobre o rendimento e qualidade de grãos. Pesq Agropec Bras, Brasília, v.18, n.3, p.219-222, 1983.

BARBOSA FILHO, M.P., DYNIA, J.F., ZIMMERMANN, F.J.P. Resposta do arroz de sequeiro ao zinco e ao cobre com efeito residual para o milho. R bras Ci Solo, Campinas, v.14, n.3, p.333-338, 1990. 
DECHEN, A.R., HAAG, H.P., CARMELLO, Q.A.C. Mecanismos de absorção e de translocação de micronutrientes In: FERREIRA, M.E., CRUZ, M.C.P. (Ed.) Micronutrientes na agricultura. Piracicaba : Potafós/CNPq, 1991. 134p, p.79-97.

DYNIA, J.F., MORAES, J.F.V. Calagem, adubação com micronutrientes e produção de arroz irrigado e feijoeiro em solo de várzea. Pesq Agropec Bras, Brasília, v.33, n.6, p.831-838, 1998

EMBRAPA. Centro Nacional de Pesquisa de Solos. Sistema brasileiro de classificação de solos. Brasília : EMBRAPA, 1999. 412p.

GALRÃO, E.Z., SOUSA, D.M.G. de., PERES, J.R.R Caracterização de deficiências nutricionais em solos de várzea da região dos cerrados. Pesq Agropec Bras, Brasília, v.19, n.9, p.1091-1101, 1984

IRGA. Estação Experimental do Arroz (Cachoerinha, RS). Arroz Irrigado: recomendações técnicas da pesquisa para o $\mathrm{Sul}$ do Brasil, 1995. 3.ed. Cachoeirinha, 1995. 88p

LOPES, M.S., SANTOS, O.S., CABRAL, J.T. et al. Efeito de micronutrientes sobre o rendimento de grãos de arroz irrigado. In: REUNIÃO DA CULTURA DO ARROZ IRRIGADO, 13, 1984, Balneário Camboriú, SC. Anais ... Florianópolis : EPAGRI, 1984. p.180-189.
MARCHEZAN, E., SANTOS, O.S., SILVA, R.P. $\boldsymbol{e}$ t $\boldsymbol{a l}$. Adubação foliar com micronutrientes em arroz irrigado, em várzea sistematizada. In: REUNIÃO DA CULTURA DO ARROZ IRRIGADO, 23, 1999, Pelotas. Anais... Pelotas : EMBRAPA Clima Temperado, 1999. p.366-368.

PAUlA, M.B. de, CARVAlHO, J.G., NOGUEIRA, F.D., $\boldsymbol{e}$ t al. Curva de resposta e avaliação de extratores para zinco disponível em solos hidromórficos e aluviais sob arroz inundado. $\mathbf{R}$ bras Ci Solo, Campinas, v.15, n.1, p.49-55, 1991.

COMISSÃO DE FERTILIDADE DO SOLO. Recomendação de adubação e de calagem para os estados do Rio Grande do Sul e de Santa Catarina. 3.ed. Passo Fundo : SBCS Núcleo Regional Sul, 1995. 223p.

REZER, J.R., MARCHEZAN, E., VIZZOTTO, V.R., $\boldsymbol{e}$ t al. Aplicação foliar de micronutrientes em arroz irrigado (Oryza sativa L.), em área de várzea sistematizada. In: REUNIÃO DA CULTURA DO ARROZ IRRIGADO, 22, 1997, Balneário Camboriú, SC. Anais... Itajaí : EPAGRI, 1997. p.248-250.

SEBRAE/RS. Diagnóstico sócio-econômico do município de São Sepé. Santa Maria : SEBRAE/FATEC, 1999. 121p.

Ciência Rural, v. 31, n. 6, 2001. 